\title{
Lung Adenocarcinoma Presenting as Malignant Pericardial Effusion/Tamponade
}

Lalitha Padmanabha Vemireddy ${ }^{1}$, Nikita Jain ${ }^{1}$, Ammar Aqeel ${ }^{1}$, Hafiz Muhammad Jeelani ${ }^{1}$, Maryna Shayuk ${ }^{1}$

1. Internal Medicine, Chicago Medical School Internal Medicine Residency Program at Northwestern McHenry Hospital, McHenry, USA

Corresponding author: Lalitha Padmanabha Vemireddy, lalitha.vemireddy@rosalindfranklin.edu

\begin{abstract}
Lung cancers are the most common primary tumors that involve the pericardium with a prevalence of up to $50 \%$. Usually, pericardial involvement goes undetected with almost $10 \%-12 \%$ found among all cancer related autopsies. Rarely pericardial effusions can be the initial site of metastasis and initial manifestation of a primary tumor. In our case, we report a 57-year-old female presenting with cardiac tamponade and subsequent testing was done which revealed lung adenocarcinoma. Malignant pericardial effusions are often silent, but certain times can present with symptoms of shortness of breath, chest pain, cough, arrhythmias, and rarely as pericardial tamponade. A high index of suspicion is required when a patient presents with tamponade to diagnose malignancy. Emergent pericardiocentesis may be warranted depending on the clinical presentation but quite often, patients tend to have a poor prognosis despite therapy given the extent of disease.
\end{abstract}

Review began 03/03/2021 Review ended 03/03/2021 Published 03/08/2021

๑) Copyright 2021

Vemireddy et al. This is an open access article distributed under the terms of the Creative Commons Attribution License CC-BY 4.0., which permits unrestricted use, distribution, and reproduction in any medium, provided the original author and source are credited.
Categories: Cardiology, Internal Medicine, Oncology

Keywords: malignant pericardial effusion, lung adenocarcinoma, tamponade

\section{Introduction}

Malignant pericardial effusion is seen more frequently with non-cardiac malignancies than primary cardiac tumors and is usually detected post mortem, with a prevalence of $10 \%-12 \%$ among all cancer related autopsies [1,2]. Although rare, it can be the initial manifestation of a primary malignancy and can sometimes present as the initial site of metastasis [1]. Lung cancer is the most common cause of pericardial involvement and studies have shown a prevalence of about $33 \%$ to $50 \%$ among all malignant pericardial effusions. Breast cancer, with a known prevalence of $18 \%$ per Strobbe et al., is the second most common cause of malignant pericardial effusion [3]. Other common tumors involved are mediastinal lymphoma, melanoma, mesothelioma, renal cell carcinoma [1,3]. Ben-Horin et al. reported an incidence of $33 \%$ (58 out of 173 patients with malignant pericardial effusions), and $22 \%$ (13 out of 59) of these patients presented with symptoms of pericardial effusion as the initial manifestation of primary malignancy [4]. Here, in this report, we present a rare case of pericardial effusion with tamponade as the initial manifestation of lung adenocarcinoma.

\section{Case Presentation}

We present a 57-year-old female with a history of hypertension who presented with worsening shortness of breath and chest pain for two days. Social history revealed that the patient was a never smoker. A review of systems was unremarkable except for bilateral leg pain with swelling. On physical examination, the patient was afebrile, tachycardic (heart rate of $127 / \mathrm{min}$ ), blood pressure of $164 / 107 \mathrm{mmHg}$, with distant heart sounds, and bilateral (b/l) calf tenderness. Initial laboratory results showed a leukocytosis of $11.3 \mathrm{k} / \mu \mathrm{L}$, brain natriuretic peptide at $45.5 \mathrm{pg} / \mathrm{ml}$, troponin $<0.04 \mathrm{ng} / \mathrm{ml}$ with the rest of the lab values being normal. Electrocardiogram on presentation showed sinus tachycardia without any ST or T wave changes or electrical alternans (Figure 1). 


\section{Cureus}

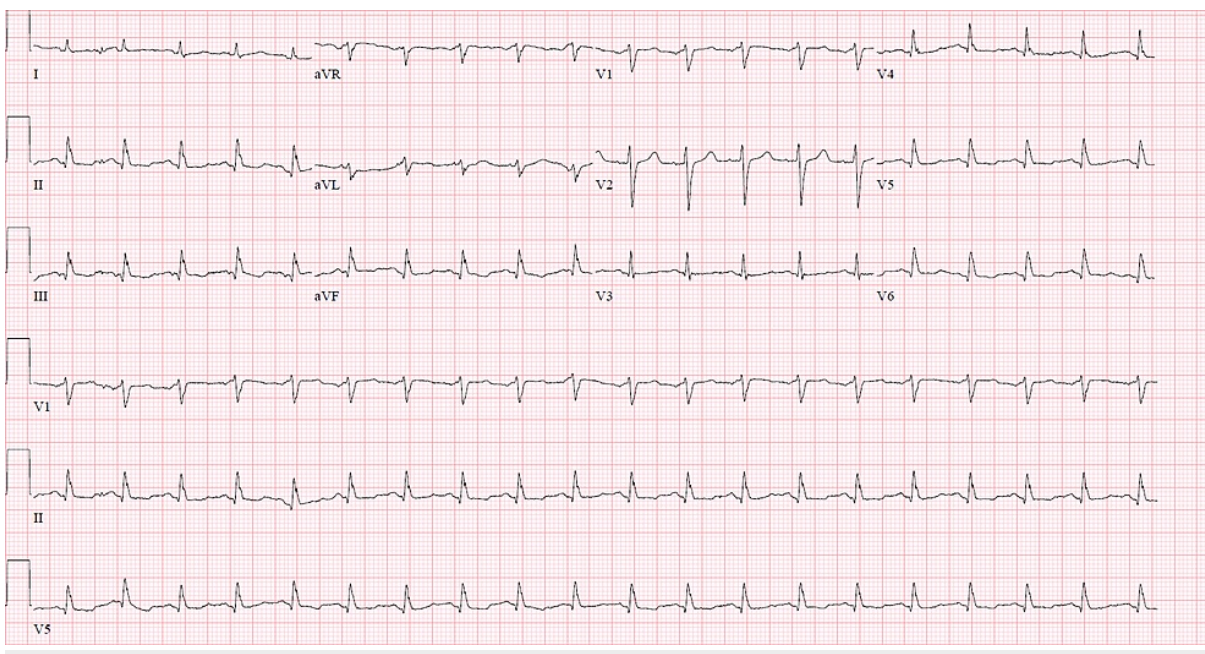

FIGURE 1: Electrocardiogram demonstrating sinus tachycardia with nonspecific ST-T segment changes.

Imaging in the emergency department included a computed tomography (CT) angiography of the chest which showed several non-occlusive pulmonary emboli in lateral basal segmental/sub-segmental and anterior basal segmental artery of left lower lobe, apical segmental artery of right upper lobe, medial segmental artery, proximal right middle lobar artery of the right middle lobe, along with a spiculated mass within the right upper lobe (Figure 2).

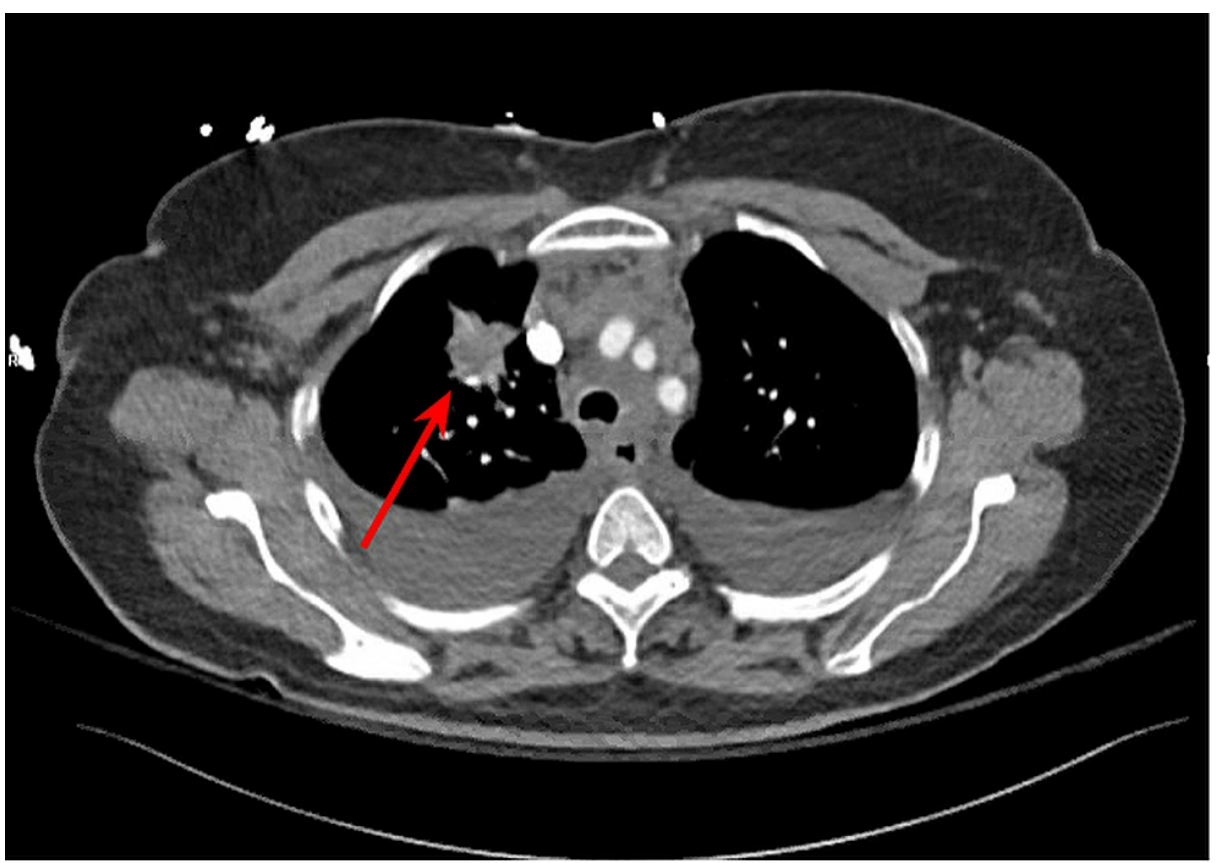

FIGURE 2: CT angiogram of the chest showing a spiculated mass in the right upper lobe (arrow).

An ultrasound of $\mathrm{b} / 1$ lower extremities showed extensive $\mathrm{b} / \mathrm{l}$ deep vein thrombosis (Figure 3). 


\section{Cureus}

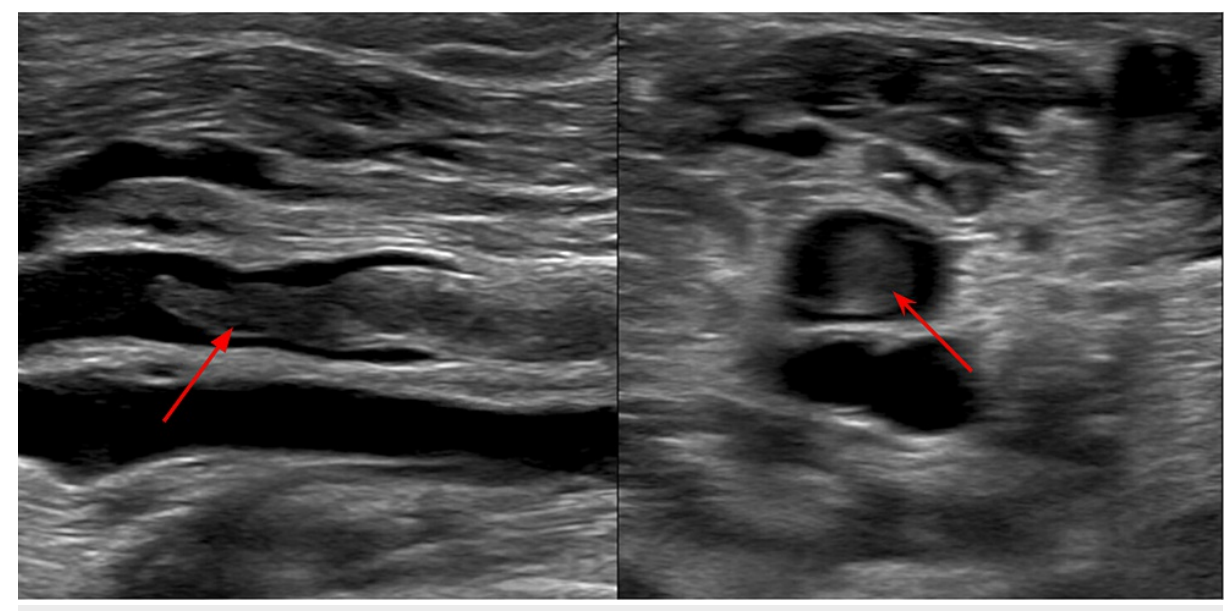

FIGURE 3: Ultrasound of the lower extremity showing thrombus in the right distal popliteal vein (arrow) in different views of ultrasound.

Echocardiogram was done to rule out right heart strain given the large burden of pulmonary embolism. It showed a large pericardial effusion with collapse of both right atrium and right ventricle (Figure 4).

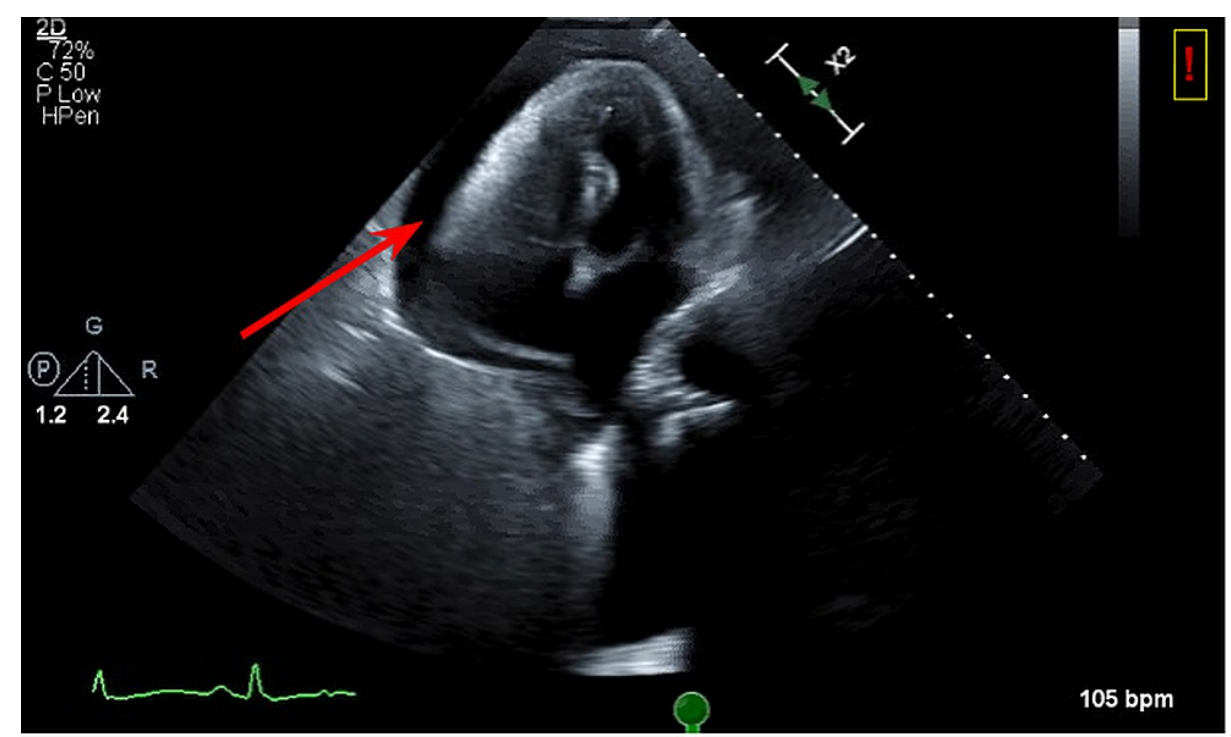

FIGURE 4: 2D echocardiogram on apical view showing fluid around the right atrium and ventricle with a completely collapsed right ventricle (arrow).

An emergent pericardiocentesis was pursued due to persistent tachycardia, distant heart sounds, and evidence of tamponade with collapse of both right atrium and ventricle. About $400 \mathrm{ml}$ of hemorrhagic pericardial fluid was removed. Analysis of the fluid revealed malignant epithelial cells consistent with metastatic lung adenocarcinoma. Pathology showed strong positivity for cytokeratin $8-18$, thyroid transcription factor-1 (TTF-1), napsin A, negative for anaplastic lymphoma kinase (ALK)/epidermal growth factor receptor (EGFR), and pending programmed death-ligand 1 (PD-L1) status (Figures 5-6). 


\section{Cureus}

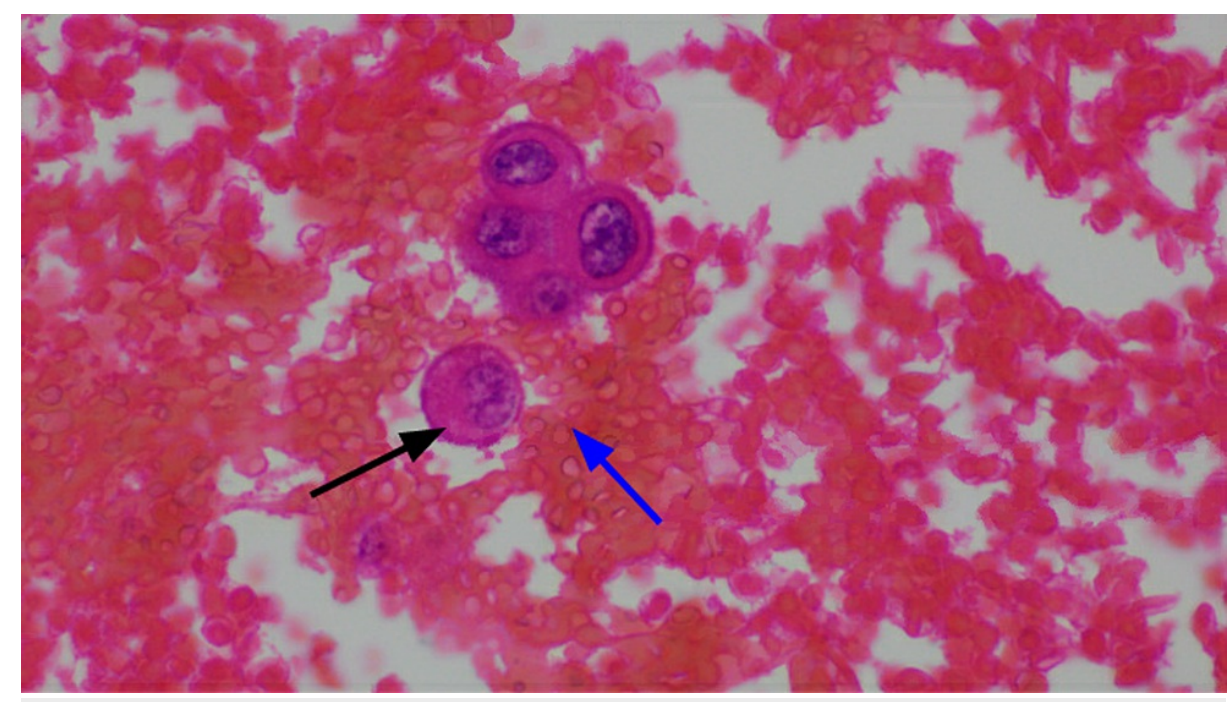

FIGURE 5: Hematoxylin and eosin staining showing the malignant adenocarcinoma cell (black arrow) with background of hemorrhagic effusion (blue arrow).

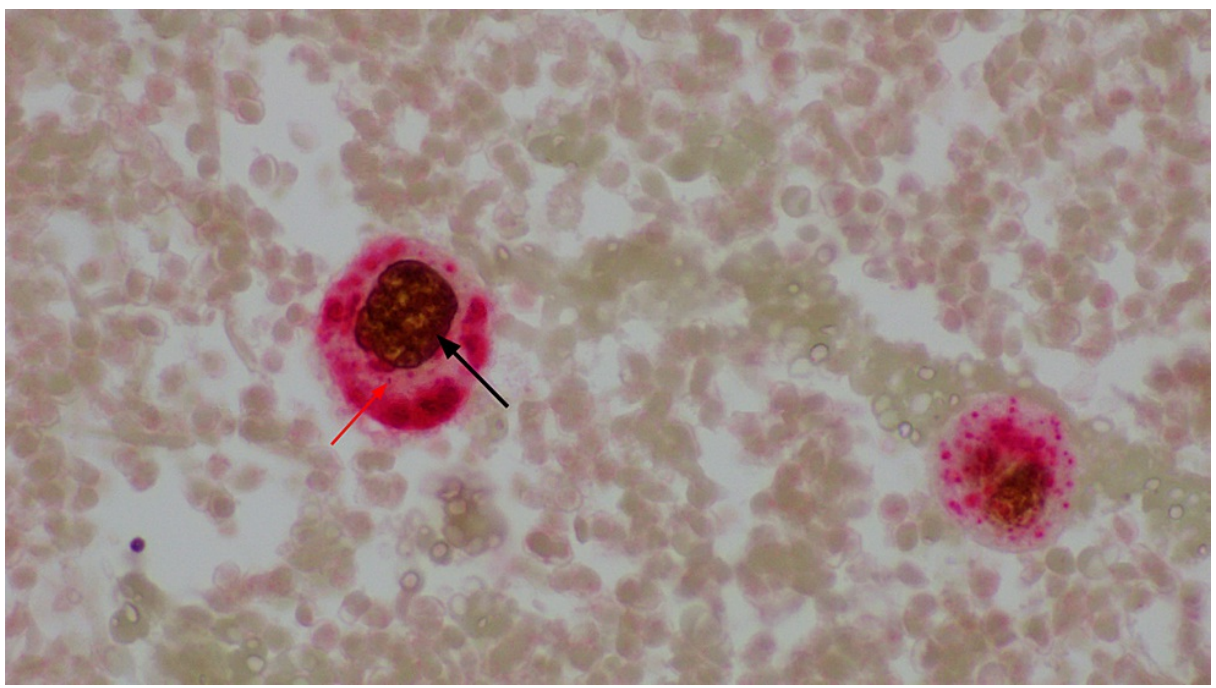

FIGURE 6: IHC showing TTF-1 (black arrow) staining of the nucleus of malignant adenocarcinoma cell of the lung with napsin-A (red arrow) staining the cytoplasm.

TTF-1: thyroid transcription factor-1; IHC: immunohistochemistry.

After pericardiocentesis, the rest of the hospital course was uneventful and the patient was discharged home. Further workup upon outpatient follow up with MRI brain showed multiple 2-5 mm enhancing lesions in the posterior fossa and supratentorial brain, consistent with metastasis (Figure 7). 


\section{Cureus}

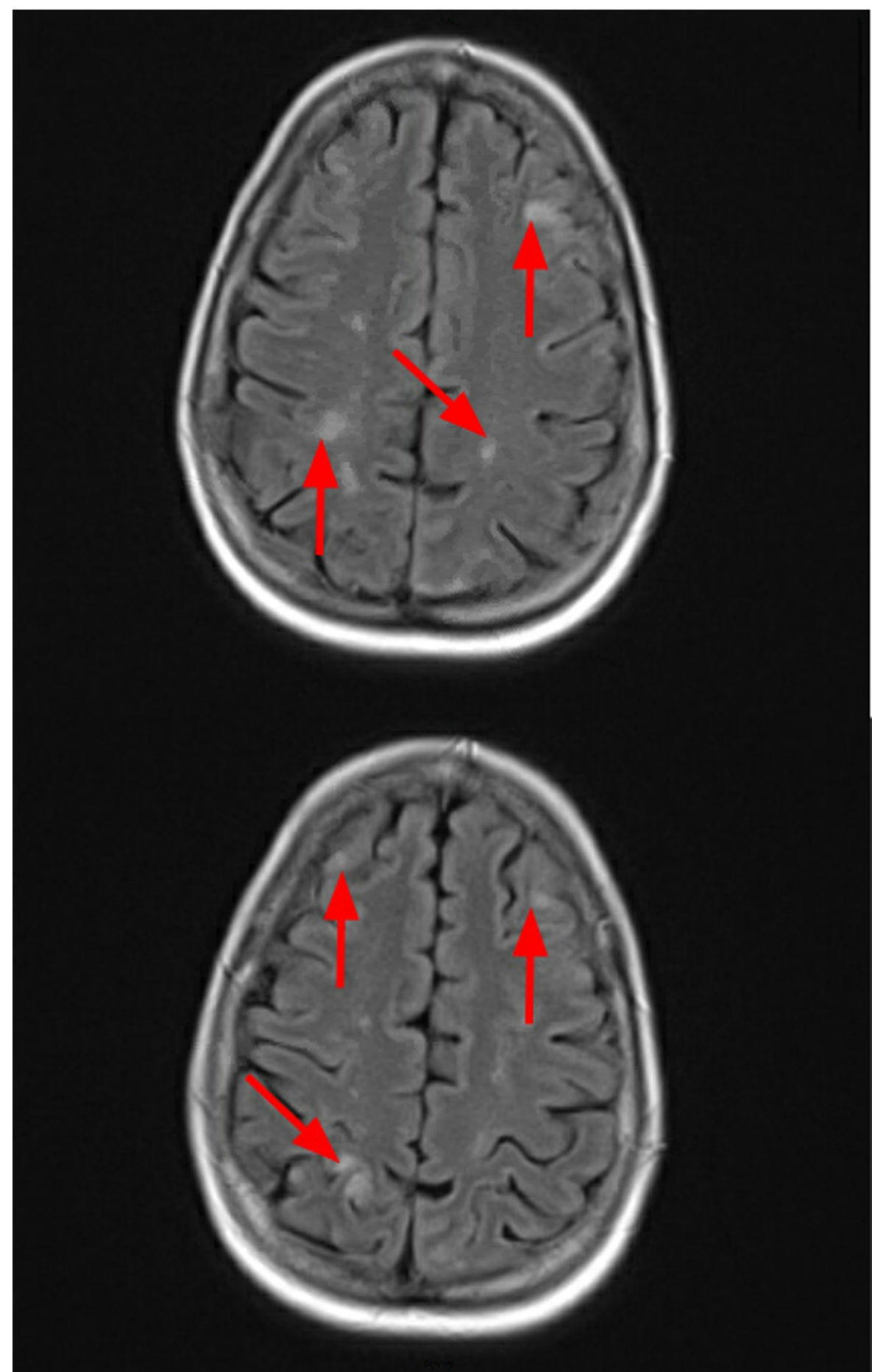

FIGURE 7: MRI brain images showing multiple lesions (arrows) in the cortex of the brain.

Patient was initiated on carboplatin, pemetrexed, and pembrolizumab for further treatment of adenocarcinoma of the lung.

\section{Discussion}

About $90 \%$ of cases of secondary cardiac tumors causing malignant pericardial effusion are clinically silent and thus, mostly found on necropsy [1]. In rare instances, patients present with symptoms such as shortness of breath, chest pain, cough, or palpitations [2,5]. In extreme cases, arrhythmias may be the initial complaint/presentation which should warrant consideration of myo-epicardial/pericardial disease involving the conduction system [2]. 
Diagnosis requires a high index of suspicion as presentation can be variable. Physical examination findings like elevated jugular venous pressure (JVP), hypotension, distant heart sounds, and pulsus paradoxus can be present and are suggestive of cardiac tamponade, but may be absent in smaller effusions [6]. Other nonspecific findings include cardiomegaly on chest radiograph, and electrical alternans or low amplitude complexes on an EKG. Echocardiography remains the initial imaging for diagnosis, showing the effusion with or without tamponade. Cardiac CT and MRI have also been used which provide a detailed view and help in differentiating the tumor from myocardium $[2,7]$. Frank tamponade and bloody effusions are more common in malignant pericardial effusion than benign causes of effusion $[5,8]$. Cytology for malignant pericardial effusion had a $51 \%$ sensitivity in a study done by Ben-Horin et al. and can range from anywhere between $66.7 \%$ to $92 \%$ based on other studies $[3,4]$.

Treatment varies based on the size of effusion and clinical presentation. In cases of tamponade with hemodynamic instability, emergent pericardiocentesis is warranted as this provides immediate relief [7,9]. In stable patients, non-emergent percutaneous or open pericardiotomy may be considered [10]. Although complication rates are lower with prolonged pericardiocentesis, the diagnostic yield and recurrence rates are similar to pericardiotomy [10]. Labbe et al. reported 2.5 months of survival rate both with pericardiocentesis and pericardiotomy [5]. Malignant pericardial effusion has a very poor prognosis especially in patients with a known history of cancer prior to presentation [3,7,11]; this is likely related to the extent of the disease.

\section{Conclusions}

Cardiac tamponade can arise secondarily from a wide range of etiologies and have varying presentations depending on the cause. Generally known to be a slowly developing and clinically silent disease process, malignant pericardial effusions can seldom present with hemodynamic instability and be the initial manifestation of an underlying malignancy. In this review, we present a rare case of metastatic lung adenocarcinoma manifesting as cardiac tamponade. If this presentation is not recognized and managed emergently, the results might be fatal, thus, it is paramount for clinicians to be aware of such atypical presentations of thoracic malignancies.

\section{Additional Information \\ Disclosures}

Human subjects: Consent was obtained or waived by all participants in this study. Conflicts of interest: In compliance with the ICMJE uniform disclosure form, all authors declare the following: Payment/services info: All authors have declared that no financial support was received from any organization for the submitted work. Financial relationships: All authors have declared that they have no financial relationships at present or within the previous three years with any organizations that might have an interest in the submitted work. Other relationships: All authors have declared that there are no other relationships or activities that could appear to have influenced the submitted work.

\section{References}

1. Shapiro LM: Cardiac tumours: diagnosis and management. Heart. 2001, 85:218-222. 10.1136/heart.85.2.218

2. Chiles C, Woodard PK, Gutierrez FR, Link KM: Metastatic involvement of the heart and pericardium: CT and MR imaging. Radiographics. 2001, 21:439-449. 10.1148/radiographics.21.2.g01mr15439

3. Strobbe A, Adriaenssens T, Bennett J, et al.: Etiology and long-term outcome of patients undergoing pericardiocentesis. J Am Heart Assoc. 2017, 6:e007598. 10.1161/JAHA.117.007598

4. Ben-Horin S, Bank I, Guetta V, Livneh A: Large symptomatic pericardial effusion as the presentation of unrecognized cancer: a study in 173 consecutive patients undergoing pericardiocentesis. Medicine. 2006, 85:49-53. 10.1097/01.md.0000199556.69588.8e

5. Labbé C, Tremblay L, Lacasse $Y$, et al.: Pericardiocentesis versus pericardiotomy for malignant pericardial effusion: a retrospective comparison. Curr Oncol. 2015, 22:412-416. 10.3747/co.22.2698

6. Sternbach G: Claude Beck: cardiac compression triads . J Emerg Med. 1988, 6:417-9. 10.1016/07364679(88)90017-0

7. Refaat MM, Katz WE.: Neoplastic pericardial effusion. Clin Cardiol. 2011, 34:593-598. 10.1002/clc.20936

8. Atar S, Chiu J, Forrester JS, Siegel RJ: Bloody pericardial effusion in patients with cardiac tamponade: is the cause cancerous, tuberculous, or iatrogenic in the 1990s?. Chest. 1999, 116:1564-1569. 10.1378/chest.116.6.1564

9. Gibbs CR, Watson RD, Singh SP, Lip GYH: Management of pericardial effusion by drainage: a survey of 10 years' experience in a city centre general hospital serving a multiracial population. Postgrad Med J. 2000, 76:809-813. 10.1136/pmj.76.902.809

10. Patel N, Rafique AM, Eshaghian S, Mendoza F, Biner S, Cercek B, Siegel RJ: Retrospective comparison of outcomes, diagnostic value, and complications of percutaneous prolonged drainage versus surgical pericardiotomy of pericardial effusion associated with malignancy. Am J Cardiol. 2013, 112:1235-1239. 10.1016/j.amjcard.2013.05.066

11. Sánchez-Enrique C, Nuñez-Gil IJ, Viana-Tejedor A, et al.: Cause and long-term outcome of cardiac tamponade. Am J Cardiol. 2016, 117:664-669. 10.1016/j.amjcard.2015.11.023 